\title{
Cost minimization for safety enhancing of timber beam structures in historical buildings
}

\author{
Anatoly Alekseytsev ${ }^{1^{*}}$, Michael Botagovsky ${ }^{2}$, Natalya Kurchenko ${ }^{2}$ \\ ${ }^{1}$ Moscow State University of Civil Engineering, Yaroslavskoe shosse, 26, Moscow, 129337, Russia \\ ${ }^{2}$ Bryansk State Engineering and Technology University, Stanke Dimitrov av., 3, Bryansk, 241037, \\ Russia
}

\begin{abstract}
A search method for finding rational parameters of beam structure bearing capacity recovery systems has been developed. This task is relevant to historic buildings containing girders with structural damage. The solution search technique is implemented by the example of calculating the parameters for timber beams external bearing capacity restoration systems with local damage or destruction of supports. An adapted genetic algorithm is used as a tool to solving the problem. A feature of this algorithm is the use of an improved random change operator and the formation of an initial and subsequent solutions sets. In this case, classical evolutionary modelling operators are not used, and the preservation of solutions from iteration to iteration is performed based on modification of the well-known elitism principle. Such computational process has allowed increasing the convergence of the iterative optimization significantly for the tasks considered. Ensuring the structure safety is achieved by strength conditions and regulating the system deflection while minimizing the costs for its operation. Herewith, the objective function allows taking into account both the lump-sum costs for system restoration arrangement and the costs for maintaining its operation during the operation cycle. An example of restoring the beam bearing capacity with the loss of stiffness of its supporting and middle span sections has been considered.
\end{abstract}

\section{Introduction}

The issues of calculation and design of timber structures are important for current studies in engineering science $[1,2]$. One of the relevant trends in this area is the repair of timber beam structures used in restoration and reconstruction of cultural heritage sites, and in restoration of bearing capacity of damaged ceiling systems and rafters with long operational cycle (Fig. 1). A significant amount of research is associated with the experimental study of the restored structures operation. Whereby, the methods of calculating their stress-strain state and the choice of rational parameters appear important. Thus, in work [3], timber beam structures are restored by using the glued rods, in article [4], for multilayer glued beams with lamination, self-tapping screws with plywood sheets are used and it is proved that the greatest effect is achieved by the preventive repair. In studies [5], wooden ceilings are restored with glued steel sheets. In some cases, steel frame prostheses $[6,7]$ are used for

\footnotetext{
*Corresponding author: aalexw@mail.ru
} 
long-term operated constructions. In this case, the maximum load on the structure is estimated experimentally. Apart from steel prostheses, reinforced concrete ones [8] also become common, which are fastened to wooden elements of gluing deformed reinforcement. Such prostheses are used to strengthen the timber frame joints. In some works, for example [9], the restoration of structures is recommended only with the use of external prosthesis, with preventive repair being preferred.

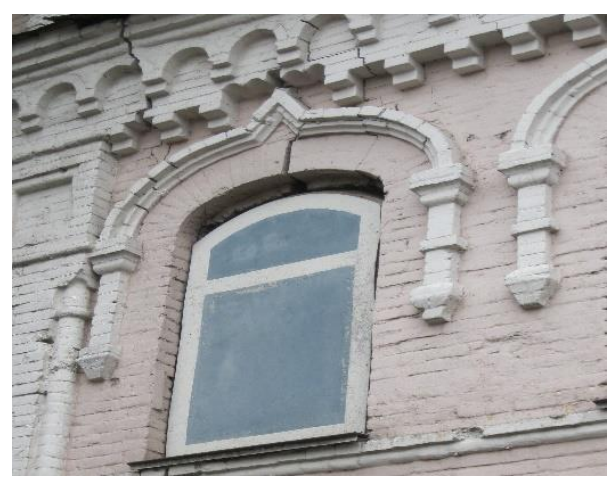

a)

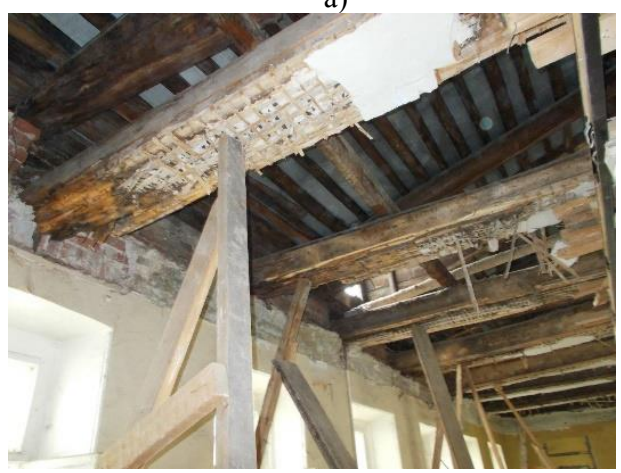

c)

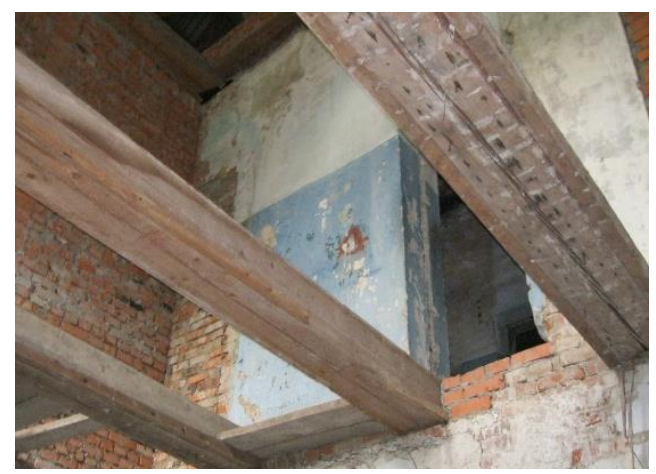

b)

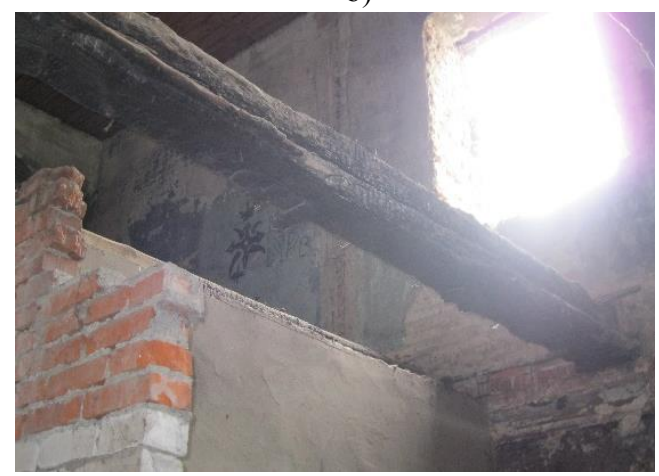

d)

Fig. 1. Specific damages of beam structures of historic buildings:

$a$ - overall view of a partial elevation in attic floor damage; $b$ - damage due to lamination from shrinkage;

$\mathrm{c}$ - decay (loss) of supporting joint; $\mathrm{d}$ - fire damage.

The issues of calculating the stress-strain state of timber systems equipped with prostheses are of great importance [7]. Three main methods have currently evolved. The first method is based on the application of the finite element methods with the possibility of the physically-nonlinear material behaviour capable of intense softening. The second is based on the application of the limit equilibrium method theorems using an elastoplastic material model that allows reproducing the brittle fracture modes quite accurately. The latter is based on the continual micromechanics suppositions with limited elastic strains. All the three methods are applied to timber structural unit models.

The issues of optimization of parameters for timber structure prosthetic systems are almost paid not enough attention in literature. This article proposes to implement such a computation scheme. The general iterative process will be based on using the adapted strategies of evolutionary modelling, described in articles [11-19]. At that, we will use a micro-genetic algorithm to select rational parameters for steel prosthetic systems. The variable parameters are represented by discrete sets of values, while the finite elements method will be used as a tool for calculating the stress-strain state. The developed technique 
will allow obtaining the design solutions of minimum cost while ensuring the strength and stiffness of the beam structures restored by applying prostheses.

\section{The formulation and method of solving the optimization problem}

Let us consider the beams or their systems that have local damage to supporting nodes or other timber parts. To restore the bearing capacity of these objects, using steel prostheses connected to intact wood structure. By doing so, the damaged wood is completely removed. The external joins of prosthesis connections are welded. In order to meet the performance requirements, preventively installed prostheses can be used, including with prestressed elements. To calculate the stress-strain state of the reconstructed object, we will use the finite element method. In this case, we will model the deformable body of the timber structure with volumetric elements, and the prosthetic system - with space rods. The prosthesis structure is topologically unchanged. To implement the optimization of the recovery system parameters, conditional minimization problem is formulated:

$$
\left(C_{r} w_{1}+C_{m} w_{2}\right) \rightarrow \min ,
$$

where $C_{r}$ is a non-recurrent (one time) expenditures, including the cost of prosthetic system arrangement; $C_{m}$ is an operating cost of the reconstructed structure under the condition of its reliability; $w_{1}, w_{2}$ is a coefficients that determine in fractions starting from one the degree of importance of non-recurrent investments and the expenditures for maintaining the system performance: $w_{1}+w_{2}=1$.

Value $C_{r}$ will be determined based on the equation:

$$
C_{r}=c_{\text {mat }} m_{\text {mat }}+c_{n} m_{n}+c_{w},
$$

where $c_{\text {mat }}, c_{n}$ is a costs per unit mass of materials of rods and node connections, respectively; $c_{w}$ is a cost of work on prosthetic system arrangement; $m_{\text {mat }}=f\left(\left\{G_{b}\right\}\right)$, is the mass of rods $; m_{n}=f\left(\left\{G_{n}\right\}\right)$ is the mass of materials for nodal connections arrangement. Cost $c_{w}$ can be approximately determined based on the analysis of analogue objects estimates as a share of costs of the sum of the first two summands in formula (2).

Element of the set $\left\{G_{b}\right\}$ for each rod varied characteristics is presented as

$$
G_{b i}=\left\{A_{i}, J_{x i}, J_{y i}, J_{z i}, \vec{e}_{i}, h_{1 i}, h_{2 i}, h_{3 i}, h_{4 i}, b_{1 i}, b_{2 i}, b_{3 i}, b_{4 i}\right\}, i \in[1 ; N],
$$

where $A_{i}$ is the area of profile chosen for $\operatorname{rod} i ; J_{x i}, J_{y i}, J_{z i}$ is a values of central inertia moments against local element axes; $\vec{e}_{i}$ is the eccentricity vector, which determines the orientation of the cross-section in the global coordinates of the finite-element model; $h_{1 i}-h_{4 i}, b_{1 i}-b_{4 i}$ is the coordinates of rod cross-sectional points where stresses are calculated; $N$ is the number of rods coinciding with the set $\left\{G_{b}\right\}$ size.

Set $\left\{G_{n}\right\}$ is presented by the assembly of elements

$$
G_{n j}=\left\{\left\{m_{l}\right\}, m_{p l}\right\}, j \in[1 ; M], l \in\left[1 ; n_{j}\right],
$$


where $m_{l}$ is a mass of weld joint per element $l$, connected in node $j$ with a gusset plate; $m_{p l}$ is a gusset plate mass; $M$ is the total number of prosthesis nodes that determines the set $\left\{G_{n}\right\}$ size; $n_{j}$ is the number of rods in node $j$.

Costs $C_{m}$ are determined by a correspondence

$$
C_{m}=\sum_{\tilde{i}=1}^{T_{m}} \frac{C_{\tilde{i}}}{(1+e)^{\tilde{i}}},
$$

where $C_{\tilde{i}}$ is a estimated operating costs (maintenance) in a $\tilde{i}$ year, $T_{m}$ is the restored structure operating period, $e$ is a coefficient of discounting. Obviously, to calculate the value of $C_{m}$, it is necessary to have, as the initial data, the data about the costs on an annual basis for maintaining the efficiency of the restored structure as set $C=\left\{C_{1}, \ldots, C_{T_{m}}\right\}$.

The equilibrium conditions of the finite-element model, the strength and stability of the prosthetic system rods, the stiffness of the structure being restored, and the prosthetic system symmetry conditions in the capacity of active constraints is tacking into account. The overall stability of the structure and local strength at the joints of the prostheses with solid (intact) timmber as passive restrictions is considered.

\section{Optimization problem solution method}

The task in hand of search type on discrete sets of the variable parameters can be solved using stochastic heuristic approaches. One of them is an evolutionary search. We will modify the algorithm with the combined constraint accounting scheme [11], modifying a number of computational procedures proposed in this paper. The variant of an object that is randomly formed using the sets $\left\{G_{b}\right\}$ of permissible values of variable parameters as an individual is considered. We will take the group of individuals considered at each step of the iterative process as a population. An elite population is a group that contains the best (1) solutions in the context of constraint satisfaction. The difference between the proposed algorithm and the one described in [11] is that the crossing-over and selection operator will not be used; instead, a random selection scheme is introduced among the first individuals of elite populations. This algorithm is called microgenetic. We will present its main stages.

1. The main population $\Pi_{A}$ by random generation by specifying an even integer $N \in[6 ; 50]$ is formed. At the same time, at this stage, elite populations containing the best solutions for $C_{r}$ and $C_{m}$, respectively, $\Pi_{B}\left(C_{r}\right)=\varnothing, \Pi_{B}\left(C_{m}\right)=\varnothing$. Population $\Pi_{A}$ is divided into two groups $\Pi_{1}$ and $\Pi_{2}$ so that $\Pi_{A}=\Pi_{1}+\Pi_{2}, \Pi_{1} \subset \Pi_{A}, \Pi_{2} \subset \Pi_{A}$, $\Pi_{1} \not \subset \Pi_{2}$. In addition, we introduce a penalty population $\Pi_{P}$, in which individuals will be placed with some minor violations of constraints.

2. The iteration process begins.

2.1 Active restrictions are verified for each individual from population $\Pi_{A}$. For these purposes, the industrial application program package solver can be used, for example, NX Nastran or Ansys complex, while the input and output data formats can be obtained textually. If restrictions are met, the individual is considered as a candidate for entry into elite populations $\Pi_{B}\left(C_{r}\right)$ и $\Pi_{B}\left(C_{m}\right)$. 
2.2 Formation of elite populations. There will be three elite populations in our proposed algorithm. First population $\Pi$ contains candidate individuals for the final decision, second $\Pi_{B}\left(C_{r}\right)$ contains the best solutions on the criterion of minimum costs for the prosthetic rod materials, third $-\Pi_{B}\left(C_{m}\right)$ contains individuals with minimal costs for the node connection arrangement.

Populations $\Pi_{B}\left(C_{r}\right), \Pi_{B}\left(C_{m}\right)$ are formed in accordance with the elitism principles: an individual is included in the population if its copy is not there, and if the target function values for it are less than the maximum value of this function for any individual in the population. Population $\Pi$ is formed based on populations $\Pi_{B}\left(C_{r}\right)$ and $\Pi_{B}\left(C_{m}\right)$. Whereby, each of the individuals of these populations is considered. In accordance with formula (1), the goal function value is calculated and the individual is included in the population, in accordance with the elitism principles.

2.3 Editing the main population. The conditions for replacement of individuals in population $\Pi_{A}$ with new individuals are as follows:

Table 1. Operators for editing elite populations.

\begin{tabular}{cc}
\hline If & Then \\
\hline$O_{i} \in \Pi_{1}$ & $\left\{\begin{array}{l}\Pi=\varnothing, \rightarrow O_{i}=\left\{\operatorname{rnd}\left(\left\{G_{b}\right\}\right)_{1}, \ldots, \operatorname{rnd}\left(\left\{G_{b}\right\}\right)_{n}\right\} \\
\Pi \neg \varnothing, \rightarrow O_{i}=O_{r n d(\bar{i})} \in \Pi\end{array}\right.$ \\
\hline$O_{i} \in \Pi_{2}$ & $O_{i}=\tilde{O}_{i} \in \Pi_{p}$ \\
\hline
\end{tabular}

where $O_{i}$ is a designation of an individual, which is a one-dimensional array with parameter codes from the set $\left\{G_{b}\right\}, \bar{i}$ is a randomly selected number identifying the number of the individual to be selected; is the size of population $\Pi$ is more than 4 , then $\bar{i}$ is a chosen randomly from the interval of whole numbers from 1 to 4 , in another case, $\bar{i}$ must not exceed the size of this population, $r n d()$ is the operator of random selection of an element from the set, $n$ is the number of variable parameters, in our particular case coinciding with the number of prosthesis rods, - an individual for which the restrictions are not satisfied.

2.4 Population $\Pi_{P}$ editing. Individuals for which restrictions are violated shall be placed in the population, in accordance with the elitism principle described above. For each individual $\tilde{O}_{i}$ the target function value is calculated, which is multiplied by the penalty coefficient $P$, according to formula (1):

$$
P=\left(\begin{array}{c}
\left.1+\chi\left(\frac{\sigma}{[\sigma]}-1\right) \cdot\left(\frac{\sigma}{[\sigma]}-1\right) k_{\sigma}+\chi\left(\frac{N_{c r}}{\left[N_{c r}\right]}-1\right) \cdot\left(\frac{N_{c r}}{\left[N_{c r}\right]}-1\right) k_{N}+\right) \\
+\chi\left(\frac{\delta}{[\delta]}-1\right) \cdot\left(\frac{\delta}{[\delta]}-1\right) k_{\delta},
\end{array}\right),
$$

where $k_{\sigma}, k_{N}, k_{\delta}$ is a coefficients that take into account the share of fine importance when the corresponding restriction is violated, $\sigma, N_{c r}, \delta$ is a maximum equivalent von 
Mises stresses in the prosthesis element, the maximum critical force for the prosthesis element, moving the restored system, respectively, $[\sigma],\left[N_{c r}\right],[\delta]$ is the permissible values of these variables; $\chi(x)$ - Heaviside function of real argument $x: \chi(x)=0$, if $x<0 ; \chi(x)=1=1$, if $x \geq 0$.

2.5 Application of mutation operator. Let the varied parameter $j$ of a certain individual $O_{i}$ have a discrete set $T_{j}$ of permissible values. This set has $w_{j}$ elements. In an interval $(0 ; 1,0)$ taking into account the normal distribution, values of random variables $m_{p}, m_{p 1}, m_{p 2}$ generate that are compared to the control numbers of $m, m_{1}, m_{2}$ mutation. With $m_{p}<m$ the number of the value in set $T_{j}$ shall be chosen randomly across the entire interval $\left[1 ; w_{j}\right]$. With condition $m_{p}<m$ cases are considered given in table 2 .

Table 2. Mutation operator content.

\begin{tabular}{ccccc}
\hline Number of value in set & \multicolumn{4}{c}{ Actions at } \\
\cline { 2 - 5 }$T_{j}$ prior to mutation & $m_{p 1}<m_{1}$ & $m_{p 1} \geq m_{1}$ & $m_{p 2}<m_{2}$ & $m_{p 2} \geq m_{2}$ \\
\hline 1 & +1 & +2 & - & - \\
2 & -1 & +1 & +2 & - \\
3 & -2 & -1 & +1 & +2 \\
4 & -2 & -1 & +1 & +2 \\
$\ldots$ & $\ldots$ & $\ldots$ & $\ldots$ & $\ldots$ \\
$w_{j}-2$ & -2 & -1 & +1 & +2 \\
$w_{j}-1$ & +1 & -1 & -2 & - \\
$w_{j}$ & -1 & -2 & - & - \\
\hline
\end{tabular}

Where the values of $-2 ;-1 ;+1 ;+2$ are whole numbers, to which size the number of the parameter value in set $T_{j}$ will be changed.

2.6 Checking the calculation stopping criteria. If at the end of $\sqrt[n / 3]{n !}$ iterations (the value is rounded to integer) there are no changes in population $\Pi$, calculations shall be ceased. The obtained result shall be considered the task solution. If there are changes in the elite population, steps 2.1-2.5 are repeated.

3. Verification of passive constraints. The variant or several variants of structures obtained as a result of the iterative process are verified by calculations using the finite element method taking into account physical and geometric nonlinearity. If all restrictions are met, a rational solution shall be considered as obtained.

\section{Optimal designing results}

Let's consider the beam system of a cultural heritage object located in Moscow (Fig. 1,c). Complete replacement of the beams is impossible. Therefore, we are considering the possibility of their prosthetics. The nature of damage to the supporting parts of the beams is shown in (Fig. 2,a). There is no significant damage in the middle parts of the beams, but there are large deflections of structures. The cross-sectional size of the beam is $450 \times 250(\mathrm{~h})$ $\mathrm{mm}$. The beam section with damaged timber, taking into account its support, is $0.5 \mathrm{~m}$. The 
beam is made of pine with an elasticity modulus of $104 \mathrm{MPa}$, a density of $560 \mathrm{~kg} / \mathrm{m}^{3}$ and a calculated bending resistance of $130 \mathrm{~kg} / \mathrm{m}^{2}$. It is required to increase the working load on the system and design the prosthetic system under normal operating conditions.

The prosthesis system consists of 2 prostheses (see Fig. 1, in pos. RT-1, RT-2), which elements are made of steel angular profiles and strips.

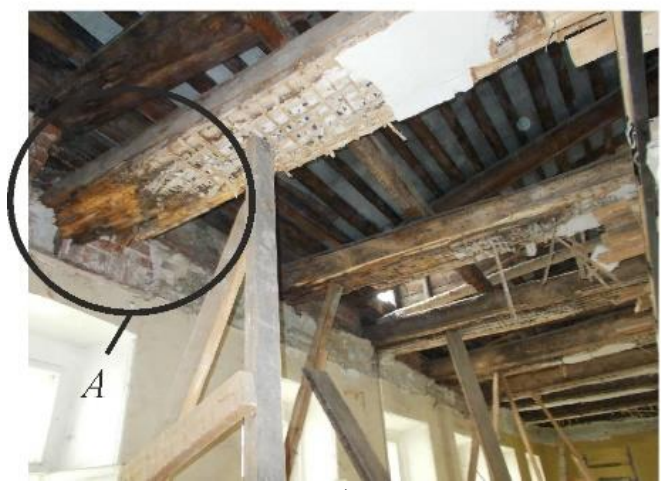

a)

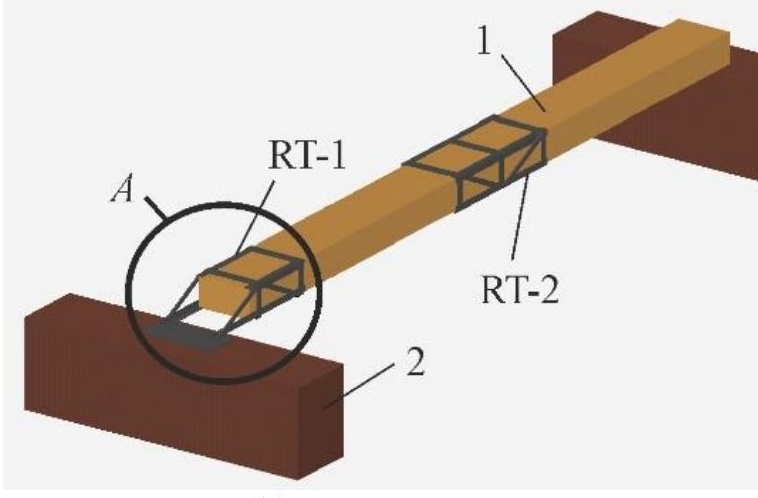

b)

Fig. 2. Objects to be restored: a - the location of roof beams and the defect of loss of support against the wall; $b$ - solid model: 1 - timber beam, 2 - supports in the form of brick walls, RT-1 support prosthetics system, RT-2 - system of preventive prosthetics, providing the beam rigidity.

The displacements of the prostheses in the horizontal direction relative to the beam are fixed with self-tapping screws. All elements of the prosthetic system are connected by welding. Structural steel C255 is used with a design bending resistance of $245 \mathrm{MPa}$. The beam span is $6 \mathrm{~m}$. The load from the weight of the roof structures in accordance with the roof operating conditions was taken to be $7.4 \mathrm{kN} / \mathrm{m}^{2}$, which corresponds to the linear uniformly distributed load of $18.5 \mathrm{kN} / \mathrm{m}^{2}$, having maximum effect on the beam, given the beam span of $2.5 \mathrm{~m}$. Torque moment effect on the beam was taken into account caused by random eccentricities of loading against its longitudinal axis. The maximum value of these eccentricities was taken to be $2-4 \mathrm{~cm}$. At that, the beam was divided into 4 equal parts along the span and the options of load location in these parts were considered taking into account the various signs of eccentricities. The beam support against the wall is hinged. We will consider the walls as rigid bodies. A solid model was developed to build a finite element model, as shown in Figure 2, b. The structure of the prostheses shown in this figure was used as the initial structure and did not change during the parametric synthesis.

The beam was schematized in a form of a volumetric body, and the prostheses were described by using three-dimensional rods. The maximum deflection of the system being restored was limited to $l / 200=3 \mathrm{~cm}$. When calculating the costs for operation (5) we took: $C_{\tilde{i}}=0,05 C_{r}(\tilde{i}-1), \widetilde{i} \in[1 ; 15], T_{m}=15, e=0.25$.

The parameters of the rods (3) varied independently for each prosthesis in accordance with the following grouping: 1) horizontal plates; 2) upper and lower angle bars; 3) vertical plates; 4) inclined plates. Within each group, the cross section of elements is assumed equal. The specification of the variable parameters is presented in Table 3. In each generation (at iteration), 50 individuals were considered. When calculating the objective function value (1) the following was assigned: $w_{1}=0.4 w_{2}=0.6$. 
Table 3. Variable profiles in rod sections.

\begin{tabular}{|c|c|}
\hline Group No. & Allowable for section selection \\
\hline 1 & 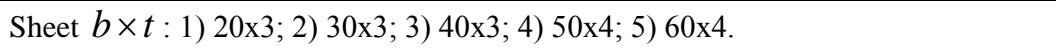 \\
\hline 2 & 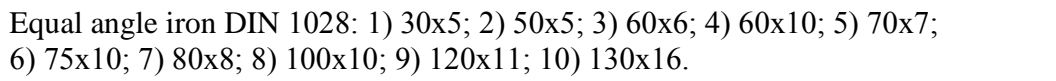 \\
\hline 3 & $\begin{array}{l}\text { Sheet } b \times t \text { : 1) } 50 \times 5 \text {; 2) } 60 \times 5 \text {; 3) } 70 \times 5 \text {; 4) } 80 \times 8 \text {; 5) } 90 \times 9 \text {; 6) } 100 \times 10 \text {; 7) } 120 \times 10 \text {; } \\
\text { 8) } 150 \times 10 \text {. }\end{array}$ \\
\hline 4 & $\begin{array}{l}\text { Sheet } b \times t \text { : 1) } 80 \times 6 \text {; 2) } 100 \times 8 \text {; 3) 100x10; 4) } 120 \times 10 \text {; 5) } 150 \times 10 \text {; 6) } 160 \times 12 \text {; } \\
\text { 7) } 180 \times 15 \text {. }\end{array}$ \\
\hline
\end{tabular}

At the same time, using only a simple single-point mutation did not allow finding solutions relatively quickly. To eliminate this problem, we used the adjustable mutation operator described in cl. 2.5 of the algorithm.

The results of task solvation are shown in Fig. 3, a-b, where the digital positions show the numbers of rational profiles from table 3. The final version was tested for meeting the passive constraints by calculating in a physically nonlinear setting by the finite element method. As a result, all set constraints were satisfied. The search process required performing not more than 150 iterations of the genetic algorithm ( 7500 calculations), while a complete enumeration of possibilities is associated with performing $4.2 \cdot 10^{7}$ object calculations. The structure deflection was $2.9 \mathrm{~cm}$; the stress in the wood of the beam was 77 $\mathrm{MPa}$. In modelling a situation, when the preventively installed prosthesis is absent in the span, the structure deflection was $5.39 \mathrm{~cm}$.

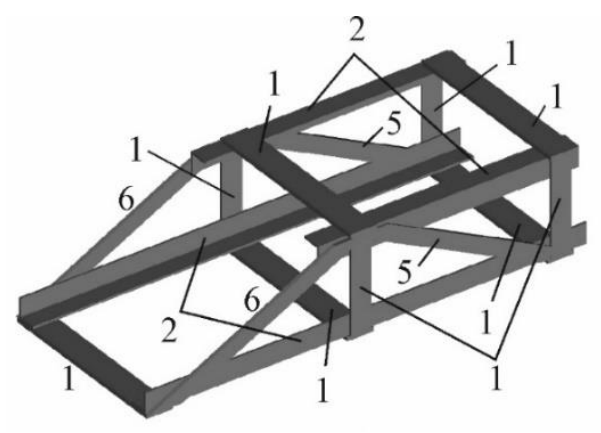

a)

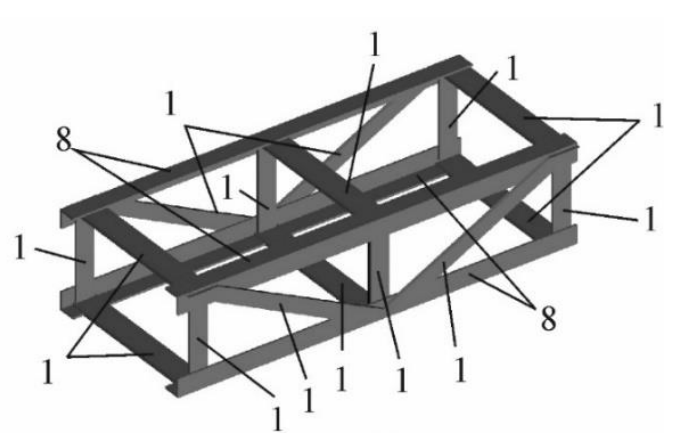

b)

Fig. 3. The results of parameter-searching for RT1 (a) and RT2 (b) systems.

\section{Discussion}

In the classical implementation of genetic algorithms, adapted for optimizing the trusses [912], selection and crossing-over operators are used. Most often, the selection is based on the roulette-wheel selection method [10], where the choice probability is inversely proportional to the mass or cost of the object. The simplest one-point scheme is introduced in the crossover operator. Calculation results showed that these operators in this task have almost no effect on the convergence. Therefore, we did not use them. In quality of prospects for further research, it is possible to note the possibility of adapting the proposed algorithm to solving the problems of preserving wooden beam structures restored by using glued rod elements, as well as to restoring the floors with plywood or steel sheets. 


\section{Conclusion}

Based on the evolutionary modelling concept, a method for finding rational parameters for beam structure bearing capacity external recovery systems has been developed. To find a solution, an adapted genetic algorithm is applied, which uses an adjustable mutation operator. The developed technique allows for ensuring the safety of the damaged structures and reducing the cost of repaired structures, taking into account the possibility of controlling the level of their deflections that can significantly increase the objects reliability.

The authors are grateful to the scientific and organizational committee of the conference for the possibility of publishing scientific research.

\section{References}

1. Kolchunov V I, Pyatikrestovskiy K P 2013 Constr. and Reconstrucr. 46 pp 25-32

2. SP 64.13330.2011. Set of rules. Timber structures. Updated version of SNiP II-25-80, 2011

3. Pizzo B, Lauriola M, 2009, Repair of timber end-beams: Some operational aspects / Protection Pro-hitech 09: 1st Int. Conf. on Protect. of Historical Buildings, Rome, Italy, 1 pp 397-402

4. Ferreira J G, Cruz H, Silva R 2017 Constr. and building mat. 154 pp 384-398

5. Pizzo B, Gavioli M, Lauriola M P 2013 Engineering Structures 48 pp 611-622

6. Jorge M B, Hélder S S, Eleftheria T 2017 Engineering Structures 140 pp 209-224

7. Füssl J, Lia M, Lukacevic M, Eberhardsteiner J, Martin C M 2017 Eng. Struct. 141 pp $427-443$

8. Metelli G, Preti M, Giuriani E 2016 Constr. and building mat. 102 pp 1018-1028

9. Negrão J H, Brito L D, Dias A G, Júnior C C, Lahr F R 2016 Constr. and building mat. 118 pp 89-103

10. Tamponea G, Ruggieri N. 2016 J. of Cultural Heritage 22 pp 1019-1027

11. Serpik I N, Alekseytsev A V, Balabin P Y 2017 Periodica Polytech. Civ. Eng. 61 pp 471-482

12. Tamrazyan A G, Avetisyan L A 2018 IOP Conf. Ser.: Mater. Sci. Eng. 365, 052034

13. Serpik I N, Alekseytsev A V 2016 Magazine of Civ Eng. 1 (61) pp 14-24

14. Alekseytsev A V, Akhremenko S A, 2018 Magazine of Civ Eng. 5 (81), 32-42

15. Serpik I N, Alekseytsev A V 2013 Magazine of Civ Eng. 9 (44) pp 23-29

16. Tamrazyan A G, Popov D 2017 MATEC Web of Conferences 11700162

17. Alekseytsev A V 2013 Magazine of Civ Eng. 5 (44) pp 28-37

18. Serpik I N, Alekseytsev A V, Balabin P Y, Kurchenko N S 2017 Magazine of Civ Eng 8 (76) pp 181-192

19. Prokurov M, Indykin A, Alekseytsev A 2018 MATEC Web of Conferences 25104017 